\title{
ASTEROSEISMOLOGY OF $\delta$ SCUTI STARS: OBSERVATIONS
}

\author{
MICHEL BREGER \\ Institut für Astronomie \\ Türkenschanzstr. 17, A-1180 Wien, Austria
}

\begin{abstract}
.
We review recent observational developments which provide important asteroseismological tools. Extensive multisite campaigns of individual $\delta$ Scuti stars show that 24 or more pulsation modes with $\ell=0$ to 2 can be detected photometrically. Spectroscopically, also about 30 modes have been detected, and these can be identified with modes of $\ell$ values up to 20 . Since each technique favors the detection of specific types of modes, hundreds or thousands of modes must be excited in $\delta$ Scuti stars.

We examine the quantities which can be matched between observations and theoretical models specifically computed for each star. Recent progress in tbe mode identification of multiple pulsation modes is illustrated by presenting an application of the phase shift method for the two stars, FG Vir and $4 \mathrm{CVn}$, recently measured by the Delta Scuti Network.
\end{abstract}

\section{Asteroseismology and $\delta$ Scuti stars}

The most promising candidates for asteroseismology on and near the main sequence are the short-period $\delta$ Scuti pulsators, which are stars with spectral types A and F and luminosity classes III-V. The key to asteroseismology is the comparison between observations and realistic stellar models. In particular, we match

- the values of the frequencies of the multiple modes together with the independently determined $(\mathrm{n}, \ell, \mathrm{m})$ quantum numbers, i. e. the identification of the pulsation modes,

- the width and position of the limited frequency range in which the pulsation modes are excited,

- the observed regularities of frequency spacing (e. g. adjacent radial orders, rotational splitting),

- the growth and decay rates through amplitude variations.

An example of applying asteroseismological tools is the predicted existence of a single, observable gravity mode for slightly evolved main sequence stars, which can 
be used to determine the star's interior rotation rate and to measure the amount of convenctive overshooting (Dziembowski \& Pamyatnykh 1991). The star CD -24 7599 is an excellent candidate to search for this mode.

Several observational and theoretical difficulties for the seismological study of $\delta$ Scuti stars need to be overcome:

- The typical $\delta$ Scuti star varies with small amplitudes of $\sim 10$ mmag. Consequently, very precise data are required to identify the individual pulsation modes.

- The excited pulsation modes are p-modes of low radial overtone, for which the asymptotic theory of frequency spacing does not apply.

- The majority of the multiperiodic $\delta$ Scuti stars are fast rotators with $v \sin i \geq$ $100 \mathrm{~km} \mathrm{~s}^{-1}$ : rapid rotation destroys the equidistant frequency spacing of the rotationally split modes that is expected for slow rotation.

- Possible differential rotation may result in a further complication of the frequency spectrum, but offers an additional motivation to apply asteroseismology to these stars.

- The multiperiodicity makes it necessary to conduct extensive multisite campaigns in order to reduce aliasing.

- Finally, most well-observed $\delta$ Scuti stars are evolved objects. The theoretical frequency spectra of such evolved objects are very dense. If correct, this increases the number of pulsation modes that need to be identified observationally as well, requiring even larger observing campaigns. (However, the extensive 1996/7 unpublished measurements by the Delta Scuti Network of the evolved star $4 \mathrm{CVn}$ have not detected this predicted dense spectrum.)

\section{New observations: oceans of nonradial modes}

The observational strategy adopted by most researchers can be summarized as follows: observationally detect the largest possible number of excited pulsation modes and identify these modes with predictions from models computed specifically for the star. During the last decade, large photometric campaigns have been very successful in detecting multiple modes and determining the frequencies. Large networks with telescopes spaced around the world are necessary in order to reduce the aliasing caused by regular day-time observing gaps and to increase the amount of information which can be collected during an observing season.

The major networks are:

- The Delta Scuti Network, which during the last few years cooperated with the Whole Earth Telescope (DSN/WET, see Breger et al. 1995, Handler 1997a). The network concentrates on long studies of a few selected stars, e.g. $300+$ hours and $20+$ detected frequencies per star. Furthermore, long-term photometric stability as well as a relative precision of 3 millimag or better per single observation is achieved by the partners. Since its inception, the network has carried out 17 multisite campaigns, which includes some shorter campaigns as well.

- STEPHI (e. g. Michel et al. 1992) and STACC Networks (Frandsen et al. 1996). Both networks specialize in studying $\delta$ Scuti stars in clusters, e. g. the variables in the Praesepe cluster. Since these $\delta$ Scuti stars in a specific cluster usually are at the same distance and share the same chemical composition, the results for different stars can be directly compared. STACC is a relatively new network using CCD detectors with high duty cycles. 


\section{Frequency $(\mu \mathrm{Hz})$}
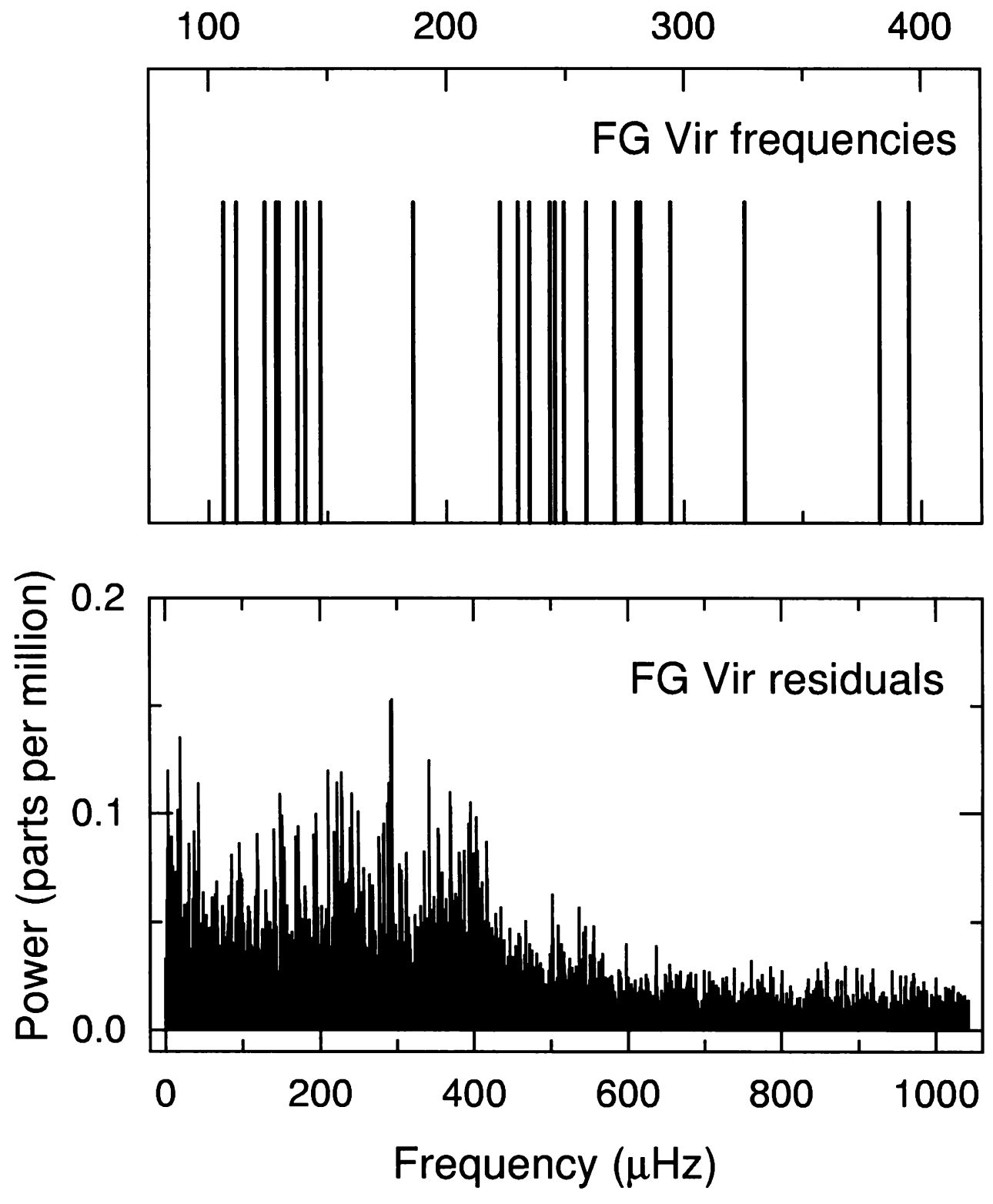

Figure 1. Pulsational behavior of FG Vir (1992-5). Top: The 24 frequencies with power signal/noise $\geq 12$. Amplitudes range from 0.0004 to 0.021 and are not shown to scale in the figure. Bottom: Power spectrum of the residuals after prewhitening the 24 frequencies. The pattern indicates that additional pulsation modes are excited in the same frequency region. 
- The Merate group (e. g. Mantegazza, Poretti \& Bossi 1996) specializes in both photometric and spectroscopic campaigns of selected stars.

The large photometric campaigns have shown that in $\delta$ Scuti stars a very large number of modes are simultaneously excited. The excitation often occurs in a very narrow frequency region. One of the $\delta$ Scuti stars with the largest number of known modes is FG Vir, for which 24 low-degree pulsation frequencies have been detected so far (Breger et al. 1997b). Fig. 1 shows the 24 frequencies of FG Vir as well as the power spectrum of the residuals. We note the sharp drop of power near $400 \mu \mathrm{Hz}$, suggesting the presence of additional excited pulsation modes shortward of $400 \mu \mathrm{Hz}$.

The photometric techniques favor the detection of low-degree modes. While these low-degree modes can also be discovered spectroscopically, (e.g. Mathias \& Aerts 1996), spectroscopy has become very important for detecting sectoral high-degree modes (e. g. Kennelly \& Walker 1996). Presently unpublished spectroscopic measurements of the star $\tau$ Peg by Kennelly, Brown and colleagues show a similar rich mode spectrum with degrees up to $\ell=20$ in a narrow frequency range. Such behavior is also confirmed by the recent results by Mantegazza (1997) for the fast rotating star V837 Cen. For this star, two prograde modes are deduced from line profiles (with $\mathrm{m}$ $=-10$ and -14 or -15 , respectively), which are not the modes seen photometrically. These high-m modes do not have the same frequency in the co-rotating frame as the low- $\ell$ modes. V837 Cen differs in this respect from $\tau$ Peg. The implication of such a narrow frequency region in terms of asteroseismology still needs to be explored.

While the majority of the observations required for asteroseismology are obtained by the major networks, important results are also published by astronomers outside these networks. Examples are the resolution of the complex behavior of $\theta$ Tuc (Paparó et al. 1996) and the eight modes determined and modelled for the star $\delta$ Scuti itself by Templeton et al. (1997).

In the next sections, we will concentrate on two stars with the largest number of photometrically determined modes: FG Vir and $4 \mathrm{CVn}$.

\section{Identification of pulsation modes}

Most successful applications of asteroseismology require that, in addition to the detection of a large number of pulsation frequencies, the actual modes of these frequencies need to be identified. This applies especially to the $\ell$ and $\mathrm{m}$ values. For $\delta$ Scuti stars, the identification of nonradial modes has been one of the most persistent problems. One of the reasons is that not all the modes in an expected pattern are excited to an observable level, e.g. of the five possible $\ell=2$ modes, only two or three might be seen. In this regard the situation is more complicated than that for PG 1159-035, for example (Winget et al. 1991). The solution lies in detecting more modes (by larger and better observing campaigns) and by applying specific mode-identification tools. The presently used methods of mode identification are:

- measure phase differences between the light curves at different wavelengths (e.g. Garrido et al. 1990),

- compare equivalent width variations of the Balmer lines with photometric or radial velocity amplitudes (Bedding et al. 1996),

- frequency values: patterns, frequency differences, $Q$ values.

For photometric campaigns carried out in at least two colors, the phase difference method is a powerful tool for mode identification. The phase shifts expected for different $\ell$ values are a function of the values of the pulsation constants $Q$ of the individual 


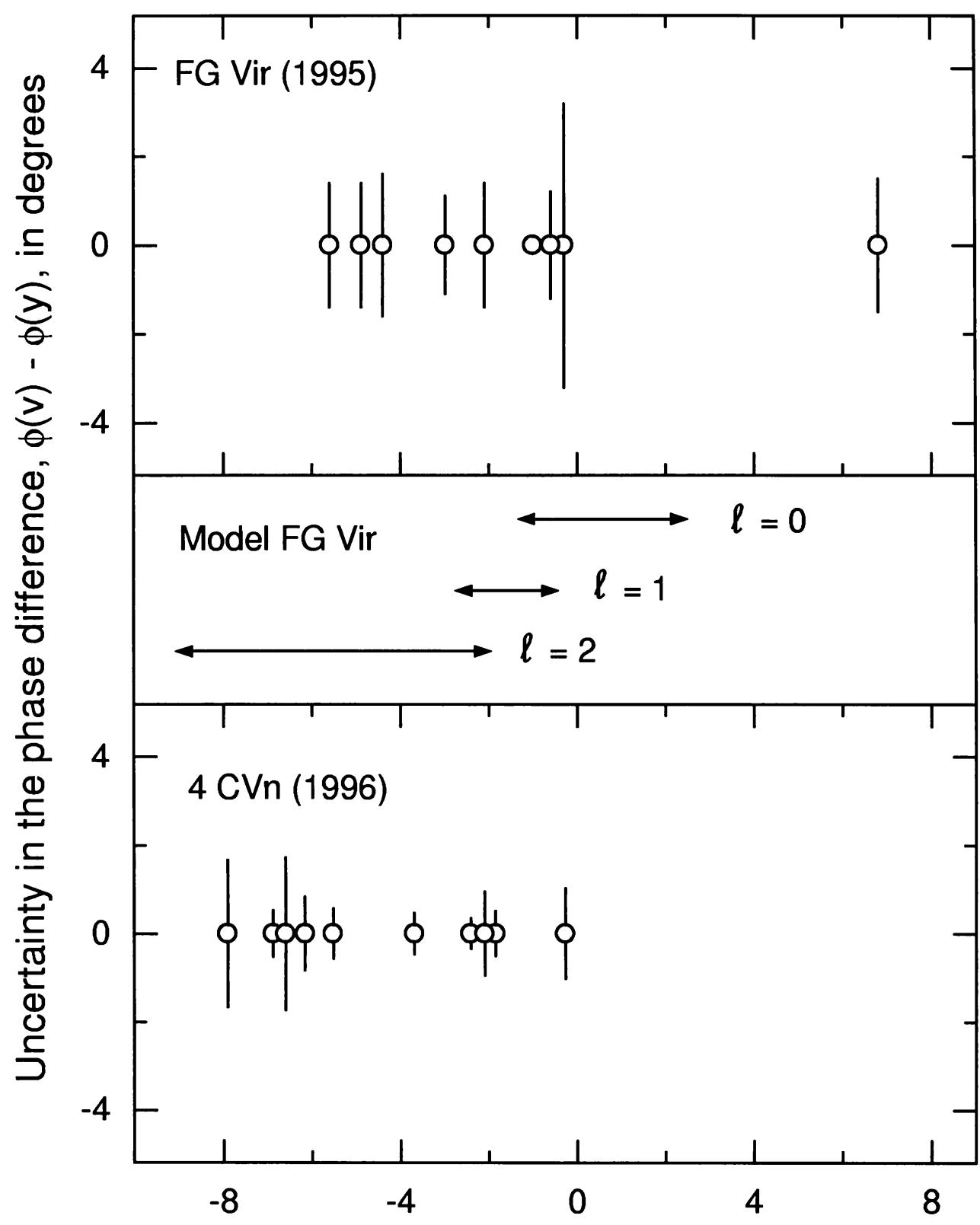

Phase difference, $\phi(v)-\phi(y)$, in degrees

Figure 2. Illustration of how photometric phase differences can be used to determine $\ell$ values. The results for FG Vir and $4 \mathrm{CVn}$ are preliminary analyses of Delta Scuti Network campaigns. The middle panel shows phase differences for different $\ell$ values covering all the different frequencies excited in FG Vir (models computed for FG Vir by R. Garrido). For individual modes, the $\ell$ determination becomes considerably less ambiguous. 


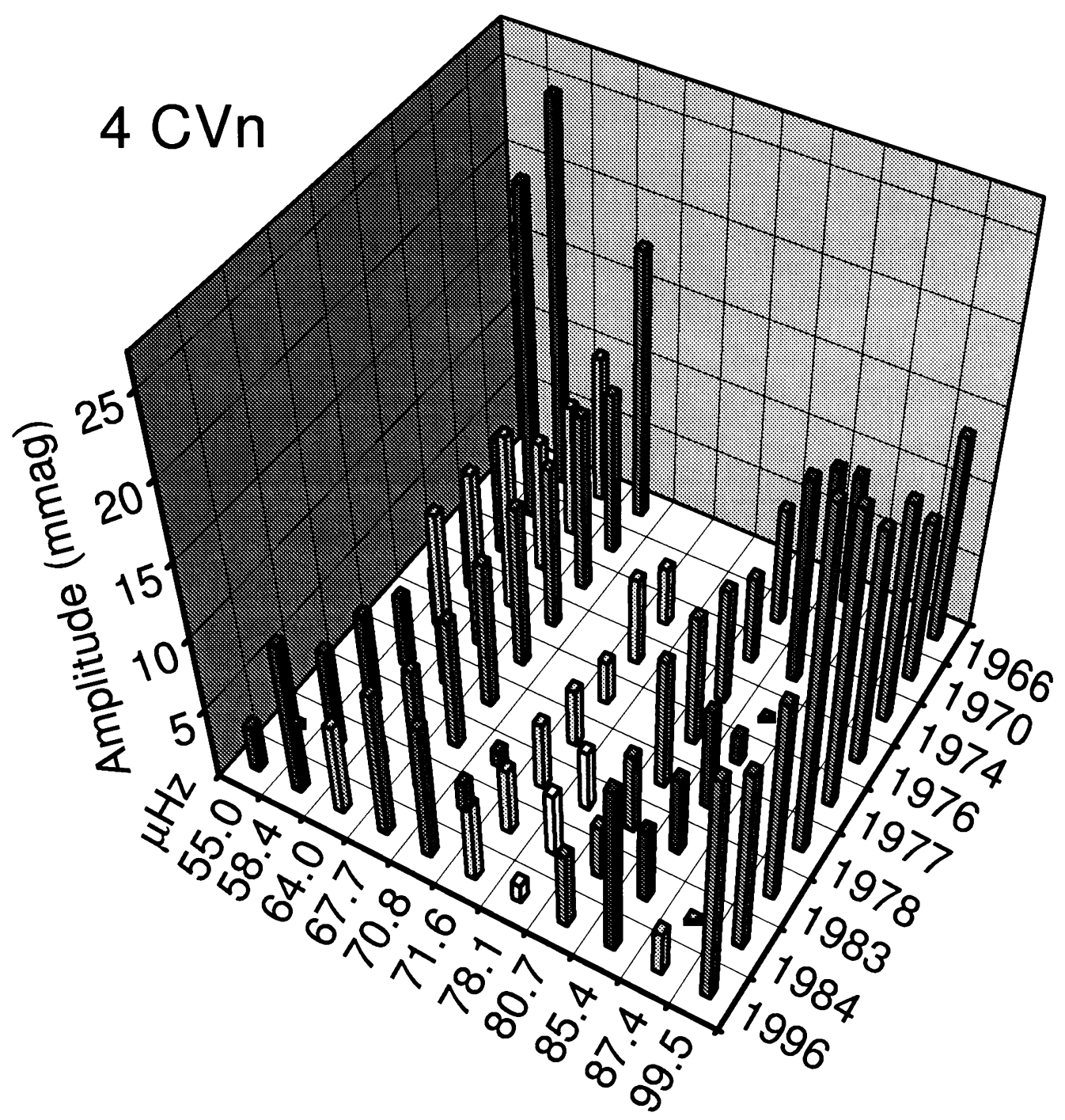

Figure 3. Amplitude variations of the major pulsation modes of $4 \mathrm{CVn}$ from 1966 to 1996. The lower axis shows the frequency of the pulsation mode plotted. Note that most amplitude variations have time scales of a decade or longer.

modes as well as the stellar parameters. The models (e.g. Garrido et al. 1990) predict both phase shifts and amplitude ratios. The work by Watson (1988) indicates that the use of the amplitude ratios still needs to be examined in more detail, since a number of stars show amplitude ratios not predicted by the models. The phase shifts, on the other hand, are less sensitive on the details of modelling and can be used. In fact, some initial successes are already obtained: 
- The phase shifts observed for the star 4 CVn by the Delta Scuti Network (unpublished data) satisfy a consistency check: in the phase shift diagram (Fig. 2), it is possible to pinpoint four adjacent pulsation modes in a central group with phase shifts near -2 to -3 degrees and the same $\ell$ value of 1 . The frequency differences between these modes are similar, viz. 14.1, 13.7 and $13.2 \mu \mathrm{Hz}$. They correspond to the expected values of four adjacent radial quantum numbers, as long as these modes have the same $\ell$ and $m$ values.

- For the star FG Vir, the mode identifications obtained by us with the phase difference method are in excellent agreement with those obtained by Viskum (1997) from the equivalent width method.

The methods of pulsation mode identification are based on the interaction between observations and theory. Despite the very encouraging initial successes, at this stage two warnings appear prudent: (i) the uncertainties of the observational results need to be kept in mind during the interpretations (and reduced in size in the future), and (ii) the theoretical models need to be further refined and individually calculated for every star.

\section{The evolved $\delta$ Scuti star 4 CVn}

During 1996 the Delta Scuti Network carried out an extensive photometric campaign of the $\delta$ Scuti variable 4 CVn (F3 III-IV) and a new neighboring $\gamma$ Doradus variable, HD 108100 at six observatories (Breger et al. 1997a). In order to increase the number of detected frequencies and to study the short-term amplitude variability, an additional campaign of $4 \mathrm{CVn}$ was carried out during 1997. While the 1997 data are not yet reduced, preliminary results for 1996 are available: 335 hours of high-quality photometry obtained during 55 nights led to the detection of 25 statistically significant frequencies of pulsation. The first results can be summarized as follows:

- 8 of the 25 detected pulsation frequencies are not independent frequencies, but combinations, $\mathrm{f}_{i} \pm f_{j}$. This number of combination frequencies is unusually large for a $\delta$ Scuti variable.

- The observed pulsation frequencies divide into three groups: in the region between 50 and $120 \mu \mathrm{Hz}$ for the excited modes, the region below $40 \mu \mathrm{Hz}$ for combination frequencies $\mathrm{f}_{i}-f_{j}$ and the region above $120 \mu \mathrm{Hz}$ for the combination frequencies $\mathrm{f}_{i}+f_{j}$.

- $4 \mathrm{CVn}$ shows a large amount of amplitude variability, while the frequencies are relatively stable (Breger 1990). It is interesting that the time scales of amplitude variability seen in the evolved star $4 \mathrm{CVn}$ are considerably longer than in the unevolved star CD -24 7599 (Handler et al. 1997b). The amplitude variability of the 11 pulsation modes with highest amplitudes are shown in Fig. 3.

- Pulsation models calculated by us with the Dziembowski code predict a dense forest of hundreds or thousands of excited frequency peaks in the power spectrum of this evolved star caused by the many g-modes from the stellar core. This would even hold for low-degree modes. This large number of excited frequencies is not seen by the observations, at least with amplitudes above the observational limit of about 0.4 millimag. In fact, the frequency spectrum resembles those of unevolved $\delta$ Scuti stars, once the frequency shift caused by the lower density is accounted for. So far, the pulsation theory used in the models cannot predict amplitudes and their variation in time. Nevertheless, the present results for $4 \mathrm{CVn}$ raise the 
tantalizing question whether there exists for evolved $\delta$ Scuti stars a presently unknown mechanism which damps pulsation in the stellar core.

During the last few years considerable progress has been made in observational asteroseismology of $\delta$ Scuti stars. With presently available equipment, the photometric observational limits can be improved relatively easily:

- The use of CCD detectors can increase the duty cycle while preserving the important low-frequency stability.

- Automatic photoelectric telescopes can considerably increase the amount of photometric data. Measurements in at least two colors can allow mode identifications. As an example, for the 1997 campaign of $4 \mathrm{CVn}$ the Vienna APT has been successfully used.

- The present limit of $10^{-4}$ in photometric amplitude can be improved with longer small-telescope campaigns of $1000+$ hours. This should raise the number of detected frequencies per star to more than 50 and allow the detection of complete multiplets of rotational splitting.

- The discovery of close frequencies in some $\delta$ Scuti stars suggests that the frequency resolution of the campaigns needs to be improved by longer coverage during the observing season. Together with other measurements from other years, a frequency resolution of $\sim 0.05 \mu \mathrm{Hz}$ should be possible.

\section{Acknowledgements}

This work has been partially supported by the Austrian Fonds zur Förderung der wissenschaftlichen Forschung, project number S7304.

\section{References}

Bedding, T. R., Kjeldsen H., Reetz, J., Barbuy B., 1996, MNRAS 280, 1155

Breger, M., 1990, A\&A 240, 308

Breger, M., Handler, G., Nather, R. E., et al., 1995, A\&A 297, 473

Breger, M., Handler, G., Garrido, R., et al., 1997a, A\&A 324, 566

Breger, M., Zima, W., Handler, G., et al. 1997b, A\&A, in press

Dziembowski, W. A., Pamyatnykh, A. A. 1991, A\&A 248, L11

Frandsen, S., Balona, L. A., Viskum, M., et al., 1996, A\&A 308, 132

Garrido, R., Garcia-Lobo, E., Rodriguez, E., 1990, A\&A 234, 262

Handler, G., Pikall, H., O'Donoghue, D., et al., 1997a, MNRAS 286, 303

Handler, G., Pamyatnykh, A. A., Zima, W., et al., 1997b, MNRAS, in press

Kennelly, E. J., Walker, G. A. H., 1996, PASP 108, 327

Mantegazza, L. 1997, A\&A 323, 844

Mantegazza, L., Poretti, E., Bossi, M., 1996, A\&A 308, 847

Mathias, P., Aerts, C., 1996, A\&A 312, 905

Michel, E., Belmonte, J. A., Alvarez, M., et al., 1992, A\&A 255, 139

Paparó, M., Sterken, C., Spoon, H. W. W., Birch, P. V., 1996, A\&A 315, 400

Templeton, M., McNamara, B. J., Guzik, J. A., 1997, AJ, in press

Viskum, M. 1997, private communication

Watson, R. D. 1988, ApSS 140, 255

Winget, D. E., Nather, R. E., Clemens, J. C., et al., 1991, ApJ 378, 326 